\title{
Alfabetización mediática de la subinformación: experiencias desde Asia
}

\section{Media literacy of subinformation: Experiences from Asia}

\author{
DOI: $10.32870 /$ mycp.v10i28.693
}

\section{Resumen}

El objetivo de este artículo es sugerir lineamientos para la alfabetización mediática sobre los procesos de subinformación, tomando como referencia la relación subinformativa de la prensa y la opinión pública respecto a las protestas prodemocráticas de 2019 de la Región Administrativa Especial de Hong Kong frente a la República Popular China. Por subinformación se entiende, a diferencia de la desinformación, la sustracción de datos esenciales en noticias publicadas por la prensa, misma que dificulta la construcción de un panorama íntegro sobre un acontecimiento de interés público. $\mathrm{Me}$ diante trabajo in situ, entrevistas en profundidad y análisis de contenido en los titulares noticiosos de la prensa más influyente en China y Hong Kong, este documento concluye que la subinformación tiene mayor presencia que la desinformación en la práctica del ejercicio periodístico, más allá de la cultura periodística.

Palabras clave: subinformación, prensa, China, Hong Kong, Asia.

\author{
Sonia Evangelina Alcántar Jaime ${ }^{1}$
}

\begin{abstract}
The objective of this article is to expose general suggestions in media literacy about the subinformative processes, taking as example the relationship between sub-information and public opinion regarding the Hong Kong Special Administrative Region pro-democratic protests and the People's Republic of China. In this document, subinformation is understood to mean, unlike misinformation, the conceals of essential data in news published by the press, which makes even more difficult to build a complete picture of a public problem. Through fieldwork, in-depth interviews, and content analysis on the most influential news headlines in China and Hong Kong, this document concludes that subinformation has a greater presence than disinformation in the practice of journalistic practice, beyond journalistic culture.
\end{abstract}

Keywords: subinformation, press, China, Hong Kong, Asia.

\section{Introducción}

En un contexto de inmediatez informativa, la prensa se desempeña como agente preponderante de la política que conlleva a la construcción de una opinión pública, es decir, influye directamente en el "conjunto de estados mentales difundidos (opinión) que interactúan con los flujos de información”

Artículo recibido el 19 de abril de 2020 y dictaminado el 6 de octubre de 2020.

1. Universidad Autónoma de Baja California. Calzada Tecnológico y Universidad S/N Delegación Mesa de Otay C.P.22390. Tijuana, Baja California, México. ORCID: https://orcid.org/0000-0002-05755125 Correo electrónico: sonia.alcantar@uabc.edu.mx 
(Sartori, 1993, p. 59). En los medios de comunicación masivos tradicionales y emergentes ${ }^{2}$ persiste una competencia por posicionar "la noticia" como narrativa dominante en los distintos medios de comunicación, misma que puede o no estar apegada a la ética del periodismo. En este aspecto, no todo lo informado a través de formato de noticia es verdad, por lo que es necesario hacer una distinción conceptual entre la noticia fabricada por la prensa y la verdad, desde la ética periodística.

Por un lado, la noticia es un relato de las condiciones sociales que puntualizan un evento y cuya información es verificable (UNESCO, 2018, p. 7), mientras que la función de la verdad es dar luz a los acontecimientos escondidos que complementen la comprensión de la lectura de la realidad política. En tal sentido, la combinación de ambos elementos en el ejercicio periodístico constituye el encuadre de una condición social que permite al individuo actuar en ella (Lippmann, 2013), es decir, crear una opinión pública.

La pendiente ascendente en los cambios de las estructuras de consumo informativo por la penetración de Internet a nivel global ha generado una compleja dinámica para la búsqueda de la verdad en la noticia, que a su vez ha transformado y diversificado el quehacer de la prensa y su análisis desde la academia. La popularidad del concepto fake news, ${ }^{3}$ españolizado como "desinformación", supone un despertar de una conciencia sobre la diversidad de información difundida a través de la prensa (UNESCO, 2018); sin embargo, en el entendido de que la desinformación se define como el proceso intencional

2. Entiéndase como medios de comunicación masivos tradicionales a la prensa, radio y televisión, antes de la aparición de Internet (y a los medios que actualmente mantienen estos formatos para la formulación y construcción de noticias). Por medios emergentes se entiende la digitalización de la noticia después de la aparición de las plataformas con Internet y nuevas formas de consumo (Boczkowsky, 2006).

3. El Programa Internacional para el Desarrollo de la Comunicación, perteneciente a la Organización de las Naciones Unidas para la Educación, la Ciencia y la Cultura (UNEsCo, por sus siglas en inglés) define las fake news como: el oxímoron que se presta para socavar la credibilidad de la información que efectivamente cumple con el umbral de verificabilidad e interés público (UNESCO, 2018, p. 7). La traducción literal de fake news es "noticias falsas". 
de tergiversar una noticia, ${ }^{4}$ existe otro elemento en el proceso periodístico que participa en la construcción de la opinión pública y del que poco se habla: la subinformación.

Por subinformación se entiende "una información totalmente insuficiente que empobrece demasiado la noticia que da, o bien, el hecho de no informar" (Sartori, 2016, p. 88); ${ }^{5}$ no significa la falsedad de una noticia, sino su reducción en exceso; este fenómeno puede ser también deliberado o no, en dependencia de: intereses políticos, líneas editoriales determinadas, la naturaleza del ejercicio periodístico, ignorancia de un acontecimiento, o falta de recursos para reportear. ${ }^{6}$ De este planteamiento surgen las siguientes preguntas: ¿cuál es el impacto de la subinformación en la opinión pública en torno al acontecer político? ¿Cómo puede reducirse la brecha de subinformación para la formación de la opinión pública integral?

En ese contexto, el objetivo de este artículo es proponer lineamientos útiles para la alfabetización mediática subinformativa tomando como referencia la exposición de la relación de la prensa entre la República Popular China y la Región Administrativa Especial de Hong Kong (HK) respecto a las protestas prodemocráticas de esta última región durante el transcurso del año 2019, con el fin de conocer la correlación entre trato subinformativo de la prensa y la opinión pública de ambas regiones. Tomar como ejemplo esta región asiática responde a que fluctúan dos sistemas políticos en un solo país, lo que polariza los modos de ejercer el periodismo de un mismo acontecimiento que concierne a ambos espacios. De esta manera, en un solo ejercicio se expone la polarización de posiciones relacionadas con la narrativa de un mismo acontecimiento.

4. La palabra desinformación tiene dos raíces etimológicas: "des" e "informar". En este caso, el prefijo "des" proviene del latín dis que significa negación, privación o acción inversa del otro componente de la palabra. Por tanto, en el entendido de que formatio es la acción de formar o dar forma para generar un producto de carácter positivo, la desinformación procura negar o privar o deformar lo que debe ser formado positivamente. En la academia (Fraguas de Pablo, 1985, 2016; Galdón, 1994; Ponsonby, 1928; Rodríguez, 2018) se sostiene que el elemento fundamental que caracteriza a la desinformación es la intencionalidad. Esto hace una diferencia conceptual entre fake news y "desinformación”. El concepto misinformation podría acercarse a la subinformación, pero en inglés es utilizado también como "desinformación", por lo tanto son distintos a la subinformación (Fox, 1983).

5. Si bien la perspectiva de Sartori responde a sus estudios en ciencias políticas, es el único investigador que ha ampliado la descripción del concepto subinformación.

6. El concepto subinformación no tiene una equivalencia en el idioma inglés: misinformation se refiere a un error en la información y disinformation a la intención de engañar; ambos conceptos se traducirían al español como "desinformación". 
Debido a la compleja dinámica en el trato informativo respecto a los temas políticos de coyuntura, metodológicamente este escrito referencia como caso paradigmático inductivo la interacción mediática en cinco de las protestas prodemocráticas ${ }^{7}$ más representativas en la escalada de violencia durante los primeros cinco meses del inicio formal de las manifestaciones de Hong Kong. Para ello se recurre al análisis de discurso titular de los sitios de noticias más influyentes en ambas regiones con líneas editoriales disímiles.

Para acompañar cualitativamente las exposiciones de la prensa sobre el acontecer político de Hong Kong, este ejercicio se complementa con trabajo in situ y entrevistas a expertos, presentando aquí las visiones de tres actores individuales: el doctor Allan Au, de la Chinese University of Hong Kong, experto en medios de comunicación en China y Hong Kong; Sheeper y Jing, dos profesionistas y líderes de las protestas. ${ }^{8}$

El desarrollo de este artículo está dividido en cuatro apartados: en la primera sección se explora y define teóricamente el concepto de subinformación y su relación con el quehacer de la prensa contemporánea. En la segunda parte se explica brevemente la situación política de Hong Kong en relación con las protestas a partir de junio 2019 y los procesos de comunicación a través de los medios de comunicación masiva tradicionales y aquéllos nacidos en Internet. Asimismo, se exponen los hallazgos cuantitativos y cualitativos del análisis de contenido sobre los enfoques titulares de la prensa online seleccionada. Finalmente, en la última sección del artículo se presenta una lista de sugerencias para aminorar los efectos de la subinformación, extraídos de los aprendizajes y experiencias desde Asia.

\section{Subinformación en la prensa contemporánea}

El concepto subinformación aplicado a las ciencias sociales fue desarrollado por la teoría crítica latinoamericana de las décadas de 1970 y 1980, definido

7. Las protestas de Hong Kong son catalogadas como prodemocráticas en este artículo porque así asumen su identidad (HK Democracy M., 2019), y así los clasifica la prensa internacional.

8. Los dos protestantes solicitaron el uso de sobrenombres por temor a represalias, ya que son líderes de las movilizaciones en la defensa de las libertades de expresión y de prensa condicionadas en las políticas básicas de la Declaración conjunta del Gobierno del Reino Unido de Gran Bretaña e Irlanda del Norte y el Gobierno de la República Popular de China sobre la cuestión de Hong Kong (Constitutional and Mainland Affairs Bureau, 1985). 
como una condición propia de esta región. ${ }^{9} \mathrm{El}$ término fue propuesto por el periodista y académico venezolano Eleazar Díaz Rangel en la publicación Pueblos Subinformados (1976), donde denunció que: 1) las agencias internacionales de noticias mantienen subinformada a una población local al describir los acontecimientos políticos internos desde una visión externa, fomentando la ignorancia de su identidad y realidad cotidiana; 2) el gobierno local no se preocupa por transparentar la producción y difusión de información, y 3) en consecuencia, el gobierno local obstruye que un pueblo cambie situaciones políticas locales que les son desfavorables. Por otro lado, tres décadas más tarde el académico y periodista José Marques de Melo (2009) retomó el concepto subinformativo aplicado a las desventajas que provienen de la existencia de una brecha digital en América Latina. En resumen, para Marques (2009), al igual que para Díaz (1976) la subinformación es una condición que deriva de las desventajas latinoamericanas en la producción y distribución de noticias, cuyas causas son múltiples.

En estas estructuras conceptuales desde las ciencias sociales, ambos autores utilizaron el término subinformación como un adjetivo calificativo propio de América Latina. ${ }^{10}$ Partiendo de esta lógica, si el participio "subinformado" se verbaliza, la prensa se transforma en un actor capaz de subinformar. En este sentido, si el adjetivo de una audiencia subinformada responde a un momento y características específicas de una región, la acción subinformativa de la prensa también podría referirse a particularidades regionales; por tanto, al articularse las características respecto a la producción, distribución y consumo informativo, pueden identificarse características subinformativas compartidas en distintas regiones del mundo.

La conversión de la subinformación de un concepto pasivo a uno activo surge de las apreciaciones de Giovanni Sartori (2016), quien separa las defi-

9. El concepto subinformativo es posicionado en los discursos políticos correspondientes al contexto histórico de los países No Alineados, durante la Conferencia de San José de Costa Rica, por la UNESCO en 1976. En las ciencias exactas el término sub-información (con un guión) ha sido utilizado en los ámbitos de patentes tecnológicas. No existe una definición teórica de la subinformación desde dicha perspectiva, pues ésta es entendida tecnológicamente en el plano digital como la sustracción de cierta información codificada en un aparato tecnológico completo.

10. Para Díaz Rangel (1976) y Marques de Melo (2009) el prefijo "sub" era relacionado en el contexto histórico de 1980, en el que se diferenciaba a los países en desarrollados y subdesarrollados. Desde esta perspectiva, quienes contaban con mayor capacidad de producción de noticias eran "superinformados", y los países con menor capacidad en la producción de noticias eran nombrados "subinformados". 
niciones desinformación y subinformación. El primer concepto estaría estrechamente vinculado al sustantivo fake news, definidas como informaciones falsas, a menudo sensacionalistas, difundidas bajo la apariencia de informes de noticias (Collins, 2017). La desinformación es definida conceptualmente por diversos académicos como la noticia cuya intención es tergiversar información y publicar noticias falseadas que provoquen engaño a la audiencia (Fraguas de Pablo, 1985, 2016; Jacquard, 1988; Ponsonby, 1928; Rodríguez, 2018; Sartori, 2016). A diferencia de la desinformación, la subinformación alude a la poca información presentada, la cual puede ser intencional o no, pero que impide conformar una integridad de la información en la noticia; por tanto, subinformación significa reducir en exceso (Sartori, 2016).

A pesar de la precisión que realiza Sartori (2016), la subinformación no es un concepto utilizado notoriamente en el campo de las ciencias sociales ni las ciencias de la comunicación, pero su práctica ha sido abordada por varias teorías del periodismo, como en el caso de la teoría instrumentalista, la teoría del agenda settings o la teoría del gatekeeper. De tal forma, la subinformación deriva a ser un concepto que puede ser estudiado como una subcategoría de la desinformación, pero también como una categoría analítica independiente con especial énfasis en la comunicación política aplicada no sólo a la región latinoamericana.

Definir qué es lo que debe informarse a través de la prensa es una tarea subjetiva, de la que Sartori (2016) traza un camino: "las noticias que vale la pena publicar son aquellas que construirán una opinión pública sobre problemas públicos de interés público" (p. 88). Por tanto, la prensa es parte actoral de la compleja estructura que decide el contenido noticioso y brinda los recursos informativos para la construcción de la opinión pública.

En el entendido de que la prensa tiene sus limitantes informativas (como los espacios en los formatos digitales e impresos, el tiempo y número de reporteros, el acceso a los líderes de instituciones públicas, entre otras), es necesario comprender que la objetividad del ejercicio periodístico no radica en el producto sino en el proceso de producción de noticias. Es decir, la producción de noticias objetivas debe cumplir con los requisitos mínimos de factualidad e imparcialidades correspondientes a la ética periodística (Lippmann, 2013; Westerståhl, 1983).

La teoría que ha abordado la práctica de subinformación (sin definirlo en ese concepto) como una rama del quehacer periodístico con especial énfasis en el ocultamiento de información, es la teoría instrumentalista de la comunicación; 
ésta afirma que "las noticias sirven objetivamente a determinados intereses políticos" (Pena de Oliveira, 2009, p. 158). Según la clasificación de Perseu Abramo respecto a esta teoría (Pena de Oliveira, 2009), existen cinco estrategias para distorsionar noticias (figura 1): las primeras tres pueden relacionarse con la desinformación: modelo de inversión, modelo de inducción, modelo global; y las últimas dos a la subinformación: modelo de ocultación, referido a la ausencia de hechos reales en la producción de la prensa; y el modelo de fragmentación, donde los acontecimientos están divididos y desconectados entre sí, de modo que se evita conciencia crítica del contexto (pp. 159 y 160). ${ }^{11}$

\section{Figura 1}

Modelo básico de características de la presentación de la noticia: información, desinformación y subinformación

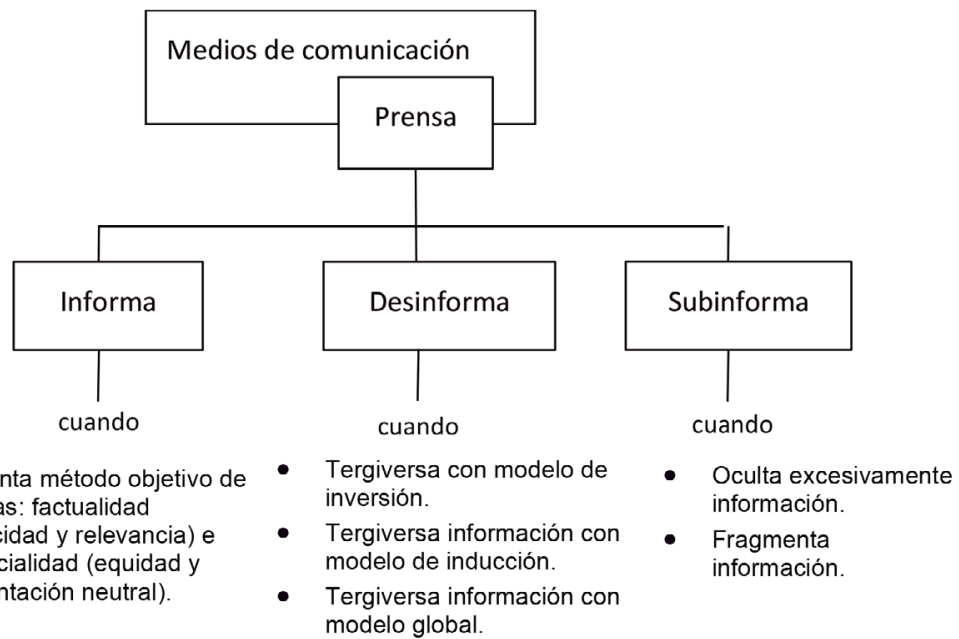

Fuente: elaboración propia basada en las características de la noticia de Lippmann (2013) y los elementos de la distorsión en la teoría instrumentalista de Perseu Abramo referido por Pena de Oliveira (2009).

11. El modelo de ocultación se refiere al intencionalmente esconder noticias (y no presentarlas, como es el caso de las fake news o desinformación que distorsionan la realidad con la noticia expuesta, la cual no cuenta con el sentido crítico de la ética periodística. La subinformación puede llegar a ocultar intencionalmente información, pues es la reducción de información o no informar. En el modelo de fragmentación, se divide la noticia y se presenta parcialmente una posición sin referir a la parte que puede ir contrario a los intereses políticos del sector que las produce. 
Cuando la prensa oculta excesivamente información valiosa o la fragmenta de tal modo que no se perciben relaciones entre las nociones del acontecer político, entonces el ejercicio periodístico subinforma a la audiencia. Al subinformar, la prensa no procura distribuir información falsa, sino que, a través de la descripción de acontecimientos verídicos, se concentra la atención en ciertos actores y hechos que ignoran la procuración de una versión más íntegra del hecho político.

El proceso de subinformación desde la prensa es un complejo entramado que va desde el trabajo del reportero hasta las líneas editoriales del periódico impreso o digital. Debido a que no existe una teoría de la subinformación desarrollada, este artículo se limita a documentar las diferencias entre el concepto de la desinformación y el de subinformación, cuyas consecuencias afectan la formulación de una opinión pública. Aunque la construcción de la opinión pública que Sartori (1993) menciona solamente aplica en democracias que presuponen las condiciones de libertad de pensamiento, libertad de expresión y policentrismo (p. 64), el esquema de la opinión pública propuesta por Lippmann (2013) abarca todo tipo de relación con la información disponible. Es decir, independientemente del sistema político que rija el flujo de información en una región determinada, los medios de comunicación ejercen un rol importante en tanto que son mediadores o voceros en la construcción de la opinión pública de la política interna o externa (Aguilar, 2017); por ende, pueden percibirse los procesos de subinformación en distintos sistemas políticos en relación con la prensa y en diferentes espacios regionales.

No es el tema de este ejercicio determinar las diferencias de los medios de comunicación en regímenes totalitarios o democracias, ni identificar si los medios de comunicación son utilizados para conseguir objetivos particulares como instrumentos de poder de Estados, instrumentos de mediación, y de comunicación entre actores (Sahagún, 1998). Lo que se pretende es identificar la actividad periodística en lo visible de la noticia publicada a través de los medios de comunicación y su relación con la opinión pública local de la República Popular China y la Región Administrativa Especial de Hong Kong, con el fin de sustraer los aprendizajes esenciales que vislumbren un primer acercamiento a la alfabetización mediática en torno a la subinformación. 


\section{Subinformación de la prensa en China y Hong Kong en lo relativo a las protestas}

A partir de junio 2019, los periódicos impresos y digitales, los canales televisivos, radiofónicos y livestreams en plataformas digitales del continente asiático viraron sus noticias al acontecer político de las protestas antigubernamentales en Hong Kong. A las marchas que al inicio solicitaban el retiro del proyecto de Ley de Extradición a China se añadieron nuevas demandas y acusaciones en contra la incidencia del Partido Comunista de China en Hong Kong (Cue, 2017, p. 88), las cuales fueron compartidas en inglés y en chino a través del portal web oficial de las protestas (HK Democracy M., 2019; HKRev-Hong Kong Local News Translation, s.f.). ${ }^{12} \mathrm{Al}$ menos una vez a la semana, jóvenes manifestantes salieron a las calles principales de Kowloon y Hong Kong Island con los emblemáticos gritos de protesta "Fight for freedom, stay with Hong Kong" y "Five demands, no one less", la cual hace referencia a las siguientes peticiones: 1) retirada absoluta del proyecto de Ley de Extradición; 2) retracción de la designación "disturbios" a las protestas; 3) amnistía para todos los manifestantes arrestados; 4) establecimiento de una comisión independiente de investigación sobre la conducta policial, y 5) doble sufragio universal para las elecciones del jefe Ejecutivo y el Consejo Legislativo.

La ausencia de un liderazgo aparente hizo notoria la diferencia entre dos grupos manifestantes: pacíficos y radicales. Ambos grupos vestían de negro y portaban máscaras para cubrir parte de su rostro; pero, mientras los primeros limitaron sus acciones a cantos, marchas, gritos y carteles en espacios públicos, los radicales rayaron paredes, obstruyeron el paso de avenidas principales, incendiaron múltiples veces entradas de las estaciones del metro, e incluso llegaron a romper los vidrios de cadenas comerciales chinas, entre otros actos vandálicos.

La mayoría de la información producida y distribuida en China y Hong Kong sobre las protestas fue a través de la prensa tradicional, aunque esta última región pudo hacerlo también a través de la prensa independiente. De acuerdo con el periodista y académico Allan Au, los sitios de noticias que más influyen en China y Hong Kong se leen en los espacios digitales y no en los periódicos o televisión, pues "la competencia está en los periódicos digitales

12. El portal de HK Democracy se encuentra al mes de septiembre 2020 en mantenimiento, por lo que a esta fecha se solicita contactarse con ellos vía Twitter. 
y lo que se comparte por Internet" (Au, 2019); sin embargo, la prensa tradicional ${ }^{13}$ también se encuentra en espacios digitales.

La China Central Television (CCTV), China Daily y la Xinhua Agency, pertenecientes al Partido Comunista de China, son los espacios online que representan la visión gubernamental de China en la producción y distribución de noticias al interior y exterior de su territorio. En Hong Kong puede accederse libremente a estos sitios de Internet en todas sus versiones de traducción lingüística. En cambio, no es posible consultar desde China continental los sitios del City Broadcasting Channel, cuya plataforma oficial es a través de Facebook, ni hay acceso a los sitios del Hong Kong Free Press, ${ }^{14}$ Radio Television Hong Kong, South China Morning Post, ni a The Standard; por tanto, la información (o subinformación) que se consume al interior de China se reduce a la información permitida por el muro de fuego chino.

Los sitios de noticias mencionados en la tabla 1 fueron los más consumidos para conocer el desarrollo de las protestas, según las apreciaciones del doctor $\mathrm{Au}$ (2019), las cuales coinciden con los activistas Sheeper (2019) y Jing (2019). La prensa que informa a través de notas periodísticas tiene valor en el consumo de información: los manifestantes entrevistados insisten en que la prensa tradicional no ha perdido valor informativo, "pero no todos son confiables" (Jing, 2019).

13. La prensa tradicional que migra a los espacios digitales sigue considerándose como "periodismo tradicional". La prensa independiente que migra a los medios de comunicación tradicionales sigue considerándose independiente por la naturaleza de su origen, más allá de su tratamiento tecnológico.

14. En su sitio web oficial, este medio se identifica y autodefine como "un periódico en inglés imparcial y sin fines de lucro. Dirigido por periodistas, respaldado por lectores y completamente independiente, HKFP se rige por un código público de ética" (Hong Kong Free Press [HKFP], 2020a). Es financiado $100 \%$ por las donaciones realizadas por voluntarios a través del portal: https:// support.hongkongfp.com/ (Hong Kong Free Press [HKFP], 2020b). 
Tabla 1

Administración de la principal prensa en China y Hong Kong en relación con las protestas prodemocráticas hongkonesas de 2019

\begin{tabular}{|c|c|c|c|c|}
\hline Nombre & Fundación & Administración & Tipo & $\begin{array}{l}\text { Sitio oficial de } \\
\text { Internet }\end{array}$ \\
\hline $\begin{array}{l}\text { China Central } \\
\text { Television (CCTV) }\end{array}$ & 1958, Pekín & $\begin{array}{l}\text { Partido } \\
\text { Comunista de } \\
\text { China }\end{array}$ & Tradicional & $\begin{array}{l}\text { http://english. } \\
\text { cctv.com/ }\end{array}$ \\
\hline China Daily & 1981, Pekín & $\begin{array}{l}\text { Partido } \\
\text { Comunista de } \\
\text { China }\end{array}$ & Tradicional & $\begin{array}{l}\text { http://www. } \\
\text { chinadaily.com. } \\
\text { cn/index.html }\end{array}$ \\
\hline $\begin{array}{l}\text { City Broadcasting } \\
(\mathrm{CBC})\end{array}$ & $\begin{array}{l}\text { 2014, Hong } \\
\text { Kong }\end{array}$ & $\begin{array}{l}\text { City University } \\
\text { of Hong Kong } \\
\text { Students' Union }\end{array}$ & Independiente & $\begin{array}{l}\text { https://www. } \\
\text { facebook.com/ } \\
\text { cityusu.cbc/ }\end{array}$ \\
\hline $\begin{array}{l}\text { Hong Kong Free } \\
\text { Press (HKFP) }\end{array}$ & $\begin{array}{l}\text { 2015, Hong } \\
\text { Kong }\end{array}$ & $\begin{array}{l}\text { Independiente: } \\
\text { Hong Kong Free } \\
\text { Press }\end{array}$ & Independiente & $\begin{array}{l}\text { https://www. } \\
\text { hongkongfp. } \\
\text { com/ }\end{array}$ \\
\hline $\begin{array}{l}\text { Radio Television } \\
\text { Hong Kong } \\
\text { (RTHK) }\end{array}$ & $\begin{array}{l}1928 \text { y } 1948, \\
\text { Hong Kong }\end{array}$ & $\begin{array}{l}\text { Oficina de } \\
\text { Comercio y } \\
\text { Desarrollo } \\
\text { Económico }\end{array}$ & Tradicional & $\begin{array}{l}\text { https://www. } \\
\text { rthk.hk/ }\end{array}$ \\
\hline $\begin{array}{l}\text { South China } \\
\text { Morning Post } \\
\text { (scMP) }\end{array}$ & $\begin{array}{l}\text { 1903, Hong } \\
\text { Kong }\end{array}$ & Alibaba Group & Tradicional & $\begin{array}{l}\text { https://www. } \\
\text { scmp.com/ }\end{array}$ \\
\hline $\begin{array}{l}\text { Xinhua News } \\
\text { Agency }\end{array}$ & 1931, Pekín & $\begin{array}{l}\text { Partido } \\
\text { Comunista de } \\
\text { China }\end{array}$ & Tradicional & $\begin{array}{l}\text { http://www. } \\
\text { xinhuanet.com// }\end{array}$ \\
\hline
\end{tabular}

Fuente: elaboración propia con base en los datos proporcionados por la página web oficial de cada medio.

Como un primer ejercicio de identificación subinformativa de la región, en este escrito se contrastan los titulares de los sitios de noticias tradicionales e independientes de Hong Kong y China respecto al desarrollo de las manifestaciones los primeros cinco meses de protesta. ${ }^{15}$ Para ello se toman como referencia los cinco días de acontecimientos coyunturales que definen parte del desarrollo de las protestas en Hong Kong:

15. Este artículo no toma como referencias los artículos de opinión de las editoriales de la prensa seleccionada, pues procura centrarse en la subinformación de la nota periodística como noticia. 
- Domingo 16 de junio. La marcha más grande registrada en la historia de Hong Kong, con la participación de dos millones de personas, lo que representa más de un cuarto de la población hongkonesa que es de casi 7.5 millones. El reporte de la policía de HK registró la presencia de 338 mil manifestantes (Vidal, 2019a).

- Lunes 1 de julio. Manifestantes irrumpen el Consejo Legislativo el día de la conmemoración del traspaso de Hong Kong a China (Westcott, 2019).

- Domingo 21 julio. Ataque en la estación del metro Yuen Long, donde al menos 45 personas fueron heridas. Los manifestantes aseguran la existencia de un vínculo entre la policía y los agresores, pues los oficiales policiacos tardaron su intervención (Blandón, 2019).

- Lunes 12 de agosto. Toma del aeropuerto y cancelación de vuelos. El día 13 de agosto se cancelaron más vuelos que el día anterior, pero este ejercicio toma como referencia el primero de los días para conocer la difusión inmediata del acontecimiento (France 24, 2019).

- Domingo 20 de octubre. El camión antidisturbios de la Policía de HK lanza líquido azul a la mezquita y otros recintos religiosos. La policía aseguró que fue contra manifestantes, pero después reconoció que fue un error (Vidal, 2019b).

Gracias al almacenamiento digital en los portales web oficiales de la prensa, hoy es posible hacer una revisión de las notas archivadas para realizar una lectura sobre las nociones de corte político a disposición de consulta (tabla 2). Al buscar información sobre Hong Kong desde China continental, los buscadores online China Daily News y Xinhua Agency bloquean las páginas específicas a las fechas de protestas masivas como la del 16 de junio, desplegando un mensaje que dice "error en la búsqueda", "no hay información disponible" o "la búsqueda contiene palabras sensibles". ${ }^{16}$

16. Esto ocurre en sus buscadores en chino, inglés y español de los mismos sitios de noticias, durante la realización de esta investigación. 


\section{Tabla 2}

Titulares de distintos diarios sobre algunas manifestaciones pro-democráticas en $\mathrm{HK}^{\mathrm{a}, \mathrm{b}}$

\begin{tabular}{|c|c|c|c|c|c|c|}
\hline \multirow[b]{2}{*}{ Fecha } & \multicolumn{3}{|c|}{ Sitios web abiertos China continental } & \multicolumn{3}{|c|}{ Sitios web cerrados en China continental } \\
\hline & $C C T V^{c}$ & China Daily & Xinhua & HKFP & RTHK & $S C M P$ \\
\hline $\begin{array}{l}16 \text { de } \\
\text { junio }\end{array}$ & $\begin{array}{l}\text { EN. HKSAR chief } \\
\text { executive announces } \\
\text { suspension of fugi- } \\
\text { tive law amendments, } \\
\text { promises to continue } \\
\text { explanation. }\end{array}$ & $\begin{array}{l}\text { No hay infor- } \\
\text { mación dis- } \\
\text { ponible en el } \\
\text { portal chino ni en } \\
\text { el portal inglés. } \\
\text { El portal suprime } \\
\text { las fechas: pasa } \\
\text { del } 15 \text { de junio al } \\
17 \text { de junio. }\end{array}$ & $\begin{array}{l}\text { CN. Hong Kong Kam Tin Mural } \\
\text { Village: } 200 \text { nominal paintings } \\
\text { of love paintings. } \\
\text { All areas of life in Hong Kong } \\
\text { support the Hong Kong SAR } \\
\text { Government to suspend the } \\
\text { revision of the Fugitive Of- } \\
\text { fenders Ordinance. }\end{array}$ & $\begin{array}{l}\text { Public anger seethes } \\
\text { in Hong Kong ahead } \\
\text { of another anti-extra- } \\
\text { dition law rally. } \\
\text { Hong Kong protesters } \\
\text { occupy roads around } \\
\text { Gov't HQ again, as } \\
\text { huge anti-extradition } \\
\text { law rally escalates. } \\
\text { Hong Kong leader } \\
\text { Carrie Lam apologizes } \\
\text { over extradition row, } \\
\text { as crowds occupy } \\
\text { roads demanding her } \\
\text { resignation. }\end{array}$ & $\begin{array}{l}\text { Carrie Lam's } \\
\text { apology not } \\
\text { enough, protest- } \\
\text { ers say. } \\
\text { CE says sorry } \\
\text { over extradition } \\
\text { saga. } \\
\text { We must fight } \\
\text { for our rights, } \\
\text { says Dennis } \\
\text { Kwok. } \\
\text { Banners ap- } \\
\text { pear on hills in } \\
\text { Kowloon and HK } \\
\text { Island. } \\
\text { Ronny Tong la- } \\
\text { ments justice has } \\
\text { lost to politics. } \\
\text { 'Loyal' officers } \\
\text { unfairly smeared: } \\
\text { police union. }\end{array}$ & $\begin{array}{l}\text { As it happened: A } \\
\text { historic day in Hong } \\
\text { Kong concludes } \\
\text { peacefully as organiz- } \\
\text { ers claim almost } 2 \\
\text { million people came } \\
\text { out in protest the } \\
\text { fugitive bill. }\end{array}$ \\
\hline
\end{tabular}




\begin{tabular}{|c|c|c|c|c|c|c|}
\hline & \multicolumn{3}{|c|}{ Sitios web abiertos China continental } & \multicolumn{3}{|c|}{ Sitios web cerrados en China continental } \\
\hline Fecha & $C C T V^{c}$ & China Daily & Xinhua & HKFP & RTHK & SCMP \\
\hline $\begin{array}{l}1 \mathrm{de} \\
\text { julio }\end{array}$ & $\begin{array}{l}\text { EN. Hong Kong marks } \\
\text { anniversary of return } \\
\text { with tech. }\end{array}$ & $\begin{array}{l}\text { EN. Lam vows to } \\
\text { give HK a new } \\
\text { start. } \\
\text { Massive rally in } \\
\text { support of Hong } \\
\text { Kong police. } \\
165,000 \text { rally for } \\
\text { city police. } \\
\text { cN. Hong Kong } \\
\text { celebrates } 22 \text { nd } \\
\text { anniversary of } \\
\text { reunification } \\
\text { flag-raising cer- } \\
\text { emony. }\end{array}$ & $\begin{array}{l}\text { EN. Hong Kong celebrates 22nd } \\
\text { anniversary of return to moth- } \\
\text { erland with various activities. } \\
\text { cN. Creativity Trend Music } \\
\text { Carnival" opens in Hong } \\
\text { Kong to showcase Hong Kong } \\
\text { achievements. } \\
\text { Yaoxiang Jiang Huan Ching } \\
\text { Chung City Achievements } \\
\text { Hong Kong 22nd Anniversary } \\
\text { Celebration Series Released. } \\
\text { Hong Kong citizens launch } \\
\text { rally to support police enforce- } \\
\text { ment. } \\
\text { Hong Kong K celebrated the } \\
\text { ceremony and the reception of } \\
\text { the flag to celebrate the 22nd } \\
\text { anniversary of the return to } \\
\text { the homeland. } \\
\text { Five thousand citizens spelled } \\
\text { "I love Hong Kong" and sent } \\
\text { a blessing to love for sAR's } \\
\text { birthday. }\end{array}$ & $\begin{array}{l}\text { 'Free Hong Kong': } \\
\text { Thousands rally for } \\
\text { democracy, as anti- } \\
\text { extradition protesters } \\
\text { occupy roads, clash } \\
\text { with police. } \\
\text { Hong Kong protest- } \\
\text { ers occupy legislative } \\
\text { chamber after smash- } \\
\text { ing windows, vandal- } \\
\text { izing corridors. } \\
\text { Organizers say } \\
\text { 550,000 attend annu- } \\
\text { al July } 1 \text { democracy } \\
\text { march as protesters } \\
\text { occupy legislature. }\end{array}$ & $\begin{array}{l}\text { Govt, allies con- } \\
\text { demn 'violent, } \\
\text { radical protest- } \\
\text { ers'. } \\
\text { UK's Hunt stress- } \\
\text { es Joint Declara- } \\
\text { tion, Hong Kong } \\
\text { autonomy. } \\
\text { HKU poll ends on } \\
\text { a low for Carrie } \\
\text { Lam. } \\
\text { Police say 'un- } \\
\text { known' fluid } \\
\text { hurled at officers. } \\
\text { Protesters } \\
\text { walked into } \\
\text { a police trap: law- } \\
\text { maker. } \\
\text { Police fired tear } \\
\text { gas in Legco } \\
\text { clearance opera- } \\
\text { tion. }\end{array}$ & $\begin{array}{l}\text { A day of unprecedent- } \\
\text { ed violence in Hong } \\
\text { Kong as protesters } \\
\text { storm the Legislative } \\
\text { Council and police fire } \\
\text { tear gas. }\end{array}$ \\
\hline
\end{tabular}

106 México y la Cuenca del Pacífico. Vol. 10, núm. 28 / enero-abril de 2021. 


\begin{tabular}{|c|c|c|c|c|c|c|}
\hline & \multicolumn{3}{|c|}{ Sitios web abiertos China continental } & \multicolumn{3}{|c|}{ Sitios web cerrados en China continental } \\
\hline Fecha & $\operatorname{CCTV}^{c}$ & China Daily & Xinhua & HKFP & RTHK & SCMP \\
\hline $\begin{array}{l}21 \mathrm{de} \\
\text { julio }\end{array}$ & $\begin{array}{l}\text { EN. Residents of } \\
\text { China's Hong Kong } \\
\text { rally to appeal for } \\
\text { peace, stability. } \\
\text { HKSAR gov't strongly } \\
\text { condemns protesters' } \\
\text { storming of liaison of- } \\
\text { fice of China's central } \\
\text { gov't. }\end{array}$ & $\begin{array}{l}316,000 \text { rally in } \\
\text { HK in support of } \\
\text { police, seek end } \\
\text { to violence. }\end{array}$ & $\begin{array}{l}\text { EN. Residents of China's Hong } \\
\text { Kong rally to appeal for peace, } \\
\text { stability. } \\
\text { CN. Hong Kong sAR govern- } \\
\text { ment spokesman strongly } \\
\text { condemns radical protesters } \\
\text { to attack central government } \\
\text { agencies in Hong Kong. }\end{array}$ & $\begin{array}{l}\text { Hong Kong braces } \\
\text { for fresh anti-gov't } \\
\text { march amid increased } \\
\text { security measures. } \\
\text { Hong Kong activists } \\
\text { hurl eggs, ink bal- } \\
\text { loons at top China } \\
\text { office, after huge } \\
\text { anti-extradition law } \\
\text { demo. } \\
\text { Hong Kong anti- } \\
\text { gov't protesters oc- } \\
\text { cupy roads and swarm } \\
\text { around legislature, } \\
\text { police HQ and China's } \\
\text { top office. } \\
\text { Hong Kong police de- } \\
\text { ploy tear gas, rubber } \\
\text { bullets against pro- } \\
\text { testers as gov't slams } \\
\text { 'direct challenge to } \\
\text { national sovereignty. }\end{array}$ & $\begin{array}{l}\text { Tens of thou- } \\
\text { sands join new } \\
\text { extradition bill } \\
\text { march. } \\
\text { Freedom, de- } \\
\text { mocracy still the } \\
\text { goals: Chu Hoi- } \\
\text { dick. } \\
\text { Security beefed- } \\
\text { up ahead of } \\
\text { Sunday's rally. } \\
\text { Marchers ignore } \\
\text { police, head } \\
\text { towards Admi- } \\
\text { ralty. } \\
\text { Riot police, pro- } \\
\text { testers play cat } \\
\text { and mouse. } \\
\text { Yuen Long MTR } \\
\text { Station closed } \\
\text { after violent } \\
\text { attacks. }\end{array}$ & $\begin{array}{l}\text { Third major march } \\
\text { against extradi- } \\
\text { tion bill turns Hong } \\
\text { Kong's financial } \\
\text { district into battle- } \\
\text { ground, sparks } \\
\text { violent attacks on } \\
\text { protesters by mob in } \\
\text { Yuen Long. } \\
\text { Protesters vandal- } \\
\text { ize Beijing's office } \\
\text { in Hong Kong after } \\
\text { hundreds of thou- } \\
\text { sands attend third } \\
\text { major march against } \\
\text { extradition bill. } \\
\text { As it happened: Hong } \\
\text { Kong police fire } \\
\text { rounds of tear gas in } \\
\text { heart of city, while } \\
\text { violence breaks out in } \\
\text { Yuen Long. } \\
\text { Rod-wielding mob } \\
\text { dressed in white } \\
\text { storms Hong Kong's } \\
\text { Yuen Long MTR sta- } \\
\text { tion, attacks protest- } \\
\text { ers and passers-by. }\end{array}$ \\
\hline
\end{tabular}




\begin{tabular}{|c|c|c|c|c|c|c|}
\hline \multirow[b]{2}{*}{ Fecha } & \multicolumn{3}{|c|}{ Sitios web abiertos China continental } & \multicolumn{3}{|c|}{ Sitios web cerrados en China continental } \\
\hline & $C C T V^{c}$ & China Daily & Xinhua & HKFP & RTHK & $S C M P$ \\
\hline $\begin{array}{l}12 \text { de } \\
\text { agosto }\end{array}$ & $\begin{array}{l}\text { EN. Stephen Perry } \\
\text { on current situa- } \\
\text { tion in HK. } \\
\text { The real truth be- } \\
\text { hind "peaceful" HK } \\
\text { protests. } \\
\text { Hong Kong celebri- } \\
\text { ties vow to protect } \\
\text { Chinese flag. }\end{array}$ & $\begin{array}{l}\text { Passengers } \\
\text { stranded at Hong } \\
\text { Kong Interna- } \\
\text { tional Airport. } \\
149 \text { arrested for } \\
\text { illegal protests, } \\
\text { violence over } \\
\text { weekend. } \\
\text { Hong Kong citi- } \\
\text { zens denounce } \\
\text { violent protests } \\
\text { HK airport au- } \\
\text { thority cancels } \\
\text { all flights for rest } \\
\text { of day. } \\
\text { Opposition } \\
\text { camp condones } \\
\text { violence so as to } \\
\text { paralyze Hong } \\
\text { Kong } \\
\text { HK govt outraged } \\
\text { by violence over } \\
\text { the weekend. }\end{array}$ & $\begin{array}{l}\text { EN. Hong Kong police arrest } \\
149 \text { people during protests in } \\
\text { last few days. } \\
\text { Liaison office of China's central } \\
\text { gov't in Hong Kong condemns } \\
\text { radical, illegal atrocities. } \\
\text { Smoothly functioning com- } \\
\text { merce in Hong Kong "funda- } \\
\text { mentally important": AmCham } \\
\text { China. } \\
\text { Hong Kong airport resumes } \\
\text { operations with over } 200 \\
\text { flights still cancelled. } \\
\text { All flights in and out Hong } \\
\text { Kong airport cancelled again. } \\
\text { One police officer seriously } \\
\text { injured by gasoline bombs in } \\
\text { Hong Kong. } \\
\text { cN. A spokesman for the HK } \\
\text { and Macao Affairs Office of } \\
\text { the State Council severely } \\
\text { condemned the attack of a } \\
\text { gasoline pump by a handful of } \\
\text { mobs in Hong Kong. } \\
\text { HK Liaison Office: Severely } \\
\text { condemns extreme illegal } \\
\text { violence. } \\
\text { Ministry of Foreign Affairs: } \\
\text { urges the United States to stop } \\
\text { intervening in HK affairs im- } \\
\text { mediately. }\end{array}$ & $\begin{array}{l}\text { Cathay Pacific warns } \\
\text { staff of dismissal for par- } \\
\text { ticipating in Hong Kong } \\
\text { 'illegal protests'. } \\
\text { 'Cruel to both sides': } \\
\text { Hong Kong protests } \\
\text { divide neighborhood } \\
\text { with police families. } \\
\text { Hong Kong police make } \\
\text { bloody arrest, assisted by } \\
\text { officers suspected to be } \\
\text { undercover as protesters. } \\
\text { Hong Kong police shoot } \\
\text { projectiles at close range } \\
\text { in Tai Koo, as protester } \\
\text { suffers ruptured eye } \\
\text { in TST. } \\
\text { China slams Hong Kong } \\
\text { protest violence as } \\
\text { 'terrorism' as People's } \\
\text { Armed Police drive } \\
\text { through neighboring } \\
\text { Guangdong. } \\
\text { All flights cancelled } \\
\text { out of Hong Kong as } \\
\text { thousands of protest- } \\
\text { ers besiege airport over } \\
\text { police violence. } \\
\text { Hong Kong police top } \\
\text { brass face barrage of } \\
\text { questions over use of } \\
\text { weapons, injuries, under- } \\
\text { cover tactics. }\end{array}$ & $\begin{array}{l}\text { Hong Kong } \\
\text { airport closure } \\
\text { casts pall over } \\
\text { markets. } \\
\text { MTR stations } \\
\text { back to normal } \\
\text { after violent } \\
\text { clashes. } \\
\text { Woman's eye } \\
\text { ruptured dur- } \\
\text { ing TST clashes: } \\
\text { sources. } \\
\text { Beijing likens } \\
\text { extradition } \\
\text { protests to ter- } \\
\text { rorism. } \\
\text { Trapped travelers } \\
\text { scramble for new } \\
\text { plans. }\end{array}$ & $\begin{array}{l}\text { Hong Kong leader } \\
\text { Carrie Lam fights } \\
\text { back tears as she } \\
\text { warns protesters, } \\
\text { they are pushing city } \\
\text { 'into an abyss'. } \\
\text { Canada's Justin } \\
\text { Trudeau extremely } \\
\text { concerned about } \\
\text { Hong Kong, urges } \\
\text { China to be careful. } \\
\text { Political solution only } \\
\text { way to return Hong } \\
\text { Kong to peace and } \\
\text { stability. } \\
\text { North Korea offers } \\
\text { support for Beijing } \\
\text { over Hong Kong } \\
\text { protests, condemning } \\
\text { 'foreign forces'. } \\
\text { Stranded passengers } \\
\text { at Hong Kong Inter- } \\
\text { national Airport. } \\
\text { Flights out of Hong } \\
\text { Kong International } \\
\text { Airport cancelled } \\
\text { as anti-government } \\
\text { protesters occupy } \\
\text { terminal building. }\end{array}$ \\
\hline
\end{tabular}

108 México y la Cuenca del Pacífico. Vol. 10, núm. 28 / enero-abril de 2021. 
Alfabetización mediática de la subinformación: experiencias desde Asia

\begin{tabular}{|c|c|c|c|c|c|c|}
\hline & \multicolumn{3}{|c|}{ Sitios web abiertos China continental } & \multicolumn{3}{|c|}{ Sitios web cerrados en China continental } \\
\hline Fecha & $C C T V^{c}$ & China Daily & Xinhua & HKFP & RTHK & SCMP \\
\hline $\begin{array}{l}20 \text { de } \\
\text { octu- } \\
\text { bre }\end{array}$ & $\begin{array}{l}\text { CN. Hong Kong trains } \\
\text { on the mainland are } \\
\text { temporarily suspend- } \\
\text { ed today. } \\
\text { EN. New Beijing } \\
\text { airport open to inter- } \\
\text { national flights. }\end{array}$ & $\begin{array}{l}\text { Lam's 3rd Policy } \\
\text { Address is inter- } \\
\text { rupted. } \\
\text { Hong Kong riot- } \\
\text { ers go on vandal- } \\
\text { ism spree. }\end{array}$ & $\begin{array}{l}\text { EN. China urges U.S. to stop in- } \\
\text { terfering in Hong Kong affairs. } \\
\text { Rioters firebomb police station, } \\
\text { vandalize facilities in renewed } \\
\text { violence in Hong Kong. } \\
\text { Hong Kong police need to use } \\
\text { appropriate force in response } \\
\text { to violence: HKSAR chief execu- } \\
\text { tive. }\end{array}$ & $\begin{array}{l}\text { Hundreds of thou- } \\
\text { sands defy protest } \\
\text { ban in Hong Kong } \\
\text { amid tear gas, vandal- } \\
\text { ism and Molotovs, as } \\
\text { mosque hit by water } \\
\text { cannon dye. } \\
\text { Hong Kong police } \\
\text { accused of targeting } \\
\text { mosque with water } \\
\text { cannon blue dye as } \\
\text { communities conduct } \\
\text { clean-up. }\end{array}$ & $\begin{array}{l}\text { Spraying of water } \\
\text { at Kowloon } \\
\text { Mosque an 'ac- } \\
\text { cident'. } \\
\text { MTR services to } \\
\text { again end at } 10 \\
\text { pm on Sunday. } \\
\text { Universal suf- } \\
\text { frage the only } \\
\text { solution: Kwok } \\
\text { Ka-ki. } \\
\text { MTR says more } \\
\text { stations to close } \\
\text { after attacks. } \\
\text { Police seize pet- } \\
\text { rol bombs, two } \\
\text { arrested. } \\
\text { Tear gas, water } \\
\text { cannon deployed } \\
\text { at Tsim Sha Tsui. }\end{array}$ & $\begin{array}{l}\text { Hong Kong leader } \\
\text { Carrie Lam pledges } \\
\text { other options if police } \\
\text { conduct probe cannot } \\
\text { quell public anger at } \\
\text { force handling of anti- } \\
\text { government protests. } \\
\text { Rampaging mobs stage } \\
\text { an arson spree in Hong } \\
\text { Kong setting mainland } \\
\text { Chinese-linked shops } \\
\text { and metro stations } \\
\text { ablaze and hurling pet- } \\
\text { rol bombs at police. } \\
\text { Political strategist Lyn- } \\
\text { ton Crosby says Hong } \\
\text { Kong government must } \\
\text { deal with issues head } \\
\text { on to resolve crisis. } \\
\text { Arrest and detention } \\
\text { of children over Hong } \\
\text { Kong's anti-govern- } \\
\text { ment protests raise } \\
\text { questions on police } \\
\text { treatment and legal } \\
\text { process. }\end{array}$ \\
\hline
\end{tabular}

a Los titulares están en inglés para reducir al mínimo las diferencias en traducción, pues la prensa señalada de HK es originalmente inglesa y la prensa china ofrece también la opción, aunque con menos información. En el caso de la prensa china, se buscó la información en ambos idiomas: se marca la diferencia con EN (English) o CN (Chinese) la secuencia del idioma en los titulares.

${ }^{\mathrm{b}}$ Ninguno de los encabezados es falso cualitativamente, pues todos pueden ser comprobados con noticias publicadas por la prensa local e internacional. ' La Televisión Central de China limita la búsqueda de noticias sobre HK en el portal chino. Solamente permite el acceso hasta 30 páginas de búsqueda de las noticias más recientes. En su portal en inglés hay más contenido.

Fuente: elaboración propia con la base de datos online de los sitios de noticias de China y Hong Kong (China Central Television, 2019; China Daily, 2019; Hong Kong Free Press, 2019; Radio Television Hong Kong, 2019; South China Morning Post, 2019; Xinhua News Agency, 2019). La búsqueda de información se realizó en octubre y noviembre de 2019 desde China y Hong Kong. 
En esta búsqueda pueden identificarse, al menos, seis tipos de posiciones desde los cuales narrar la noticia: 1) ciudadanos hongkoneses a favor de la paz y acciones policiacas; 2) participantes de protestas pacíficas; 3) participantes de protestas radicales y sus afectaciones públicas; 4) agresiones policiales y de mafias en contra de protestantes; 5) declaraciones gubernamentales específicas sobre las protestas, y 6) aquellos pronunciamientos de otros gobiernos y empresas sobre temas relacionados con las protestas. A continuación, en la tabla 3 se resume cuantitativamente el contenido de los titulares de noticias según el enfoque designado en las notas periodísticas en mi propuesta de categoría de posiciones de la noticia. 
Alfabetización mediática de la subinformación: experiencias desde Asia

Tabla 3

Análisis cuantitativo de enfoques noticiosos de la prensa china y hongkonesa

\begin{tabular}{|c|c|c|c|c|c|c|c|c|c|c|c|c|c|c|}
\hline \multirow[b]{2}{*}{ Posiciones de la noticia } & \multicolumn{2}{|c|}{ CCTV } & \multicolumn{2}{|c|}{$C D$} & \multicolumn{2}{|c|}{ Xinhua } & \multirow{2}{*}{$\begin{array}{c}\text { Fuentes } \\
\text { chinas } \\
\%\end{array}$} & \multicolumn{2}{|c|}{ HKFP } & \multicolumn{2}{|c|}{ RTHK } & \multicolumn{2}{|c|}{$S C M P$} & \multirow{2}{*}{$\begin{array}{c}\begin{array}{c}\text { Fuentes } \\
\text { hongkonesas }\end{array} \\
\%\end{array}$} \\
\hline & $\#$ & $\%$ & $\#$ & \% & $\#$ & $\%$ & & $\#$ & $\%$ & $\#$ & $\%$ & $\#$ & $\%$ & \\
\hline $\begin{array}{l}\text { Hongkoneses en petición por } \\
\text { paz y apoyo a la policía }\end{array}$ & 1 & 11.1 & 4 & 33.3 & 3 & 15.8 & 20.1 & 0 & 0.0 & 0 & 0.0 & 0 & 0.0 & 0.0 \\
\hline $\begin{array}{l}\text { Protestas pro-democráticas } \\
\text { pacíficas }\end{array}$ & 0 & 0.0 & 0 & 0.0 & 0 & 0.0 & 0.0 & 4 & 19.0 & 2 & 7.1 & 1 & 6.3 & 10.8 \\
\hline $\begin{array}{l}\text { Protestas pro-democráticas } \\
\text { radicales y afectaciones públicas }\end{array}$ & 2 & 22.2 & 4 & 33.3 & 4 & 21.1 & 25.5 & 6 & 28.6 & 11 & 39.3 & 4 & 25.0 & 31.0 \\
\hline $\begin{array}{l}\text { Agresiones policiales y mafias } \\
\text { contra protestantes }\end{array}$ & 0 & 0.0 & 0 & 0.0 & 0 & 0.0 & 0.0 & 6 & 28.6 & 2 & 7.1 & 6 & 37.5 & 24.4 \\
\hline $\begin{array}{l}\text { Declaraciones gubernamentales } \\
\text { y de la policía sobre protestas }\end{array}$ & 2 & 22.2 & 2 & 16.7 & 4 & 21.1 & 20.0 & 3 & 14.3 & 9 & 32.1 & 1 & 6.3 & 17.6 \\
\hline $\begin{array}{l}\text { Declaraciones de gobierno, } \\
\text { policiales y empresariales } \\
\text { relacionadas a las protestas }\end{array}$ & 1 & 11.1 & 2 & 16.7 & 1 & 5.3 & 11.0 & 2 & 9.5 & 4 & 14.3 & 4 & 25.0 & 16.3 \\
\hline Otros temas & 3 & 33.3 & 0 & 0.0 & 7 & 36.8 & 23.4 & 0 & 0.0 & 0 & 0.0 & 0 & 0.0 & 0.0 \\
\hline TOTAL & 9 & 100 & 12 & 100 & 19 & 100 & 100 & 21 & 100 & 28 & 100 & 16 & 100 & 100 \\
\hline
\end{tabular}

Elaboración propia con la base de datos de la tabla 2. 
Los hallazgos expuestos en la tabla 3 exponen que los sitios de noticias ССтV, China Daily y Xinhua Agency etiquetan a todas las manifestaciones como radicales y causantes de perjuicio de los espacios públicos, al hacer uso recurrente de los adjetivos calificativos "vandalismo" y "violencia" en sus titulares (véanse columnas 2, 3 y 4 de la tabla 2). El 40\% del contenido expone que la población y el Gobierno hongkonés, al igual que el Gobierno chino, están "en contra de la violencia", por tanto, de las manifestaciones en general, pues en el $25 \%$ de su contenido refuerzan discursivamente que las manifestaciones están relacionadas con un nivel de agresividad. Además, en los titulares ignoran (no informan) los métodos policiales antidisturbios y en $23.4 \%$ comparten otras noticias sobre Hong Kong no relacionadas con las protestas.

Por otro lado, los medios locales hongkoneses - dos de ellos tradicionales y uno independiente-, lejos de favorecer con titulares a las manifestaciones pacíficas, ocupan un $31 \%$ de su contenido en los actos de violencia y vandalismo de los protestantes radicales, un $6 \%$ más que los medios de China continental. Sin embargo, equilibra la descripción de actos por parte de los manifestantes radicales con las agresiones de la policía y otros actores como la mafia en Yuen Long en contra de los protestantes en casi el 25\% de sus titulares. Y, a diferencia de los medios chinos, la HKFP, RTHK y SCMP muestran declaraciones gubernamentales sobre las protestas en Hong Kong de posturas a favor y en contra de la lucha antigubernamental (véanse columnas 5, 6 y 7 de la tabla 2).

Todos los titulares seleccionados para este ejercicio tienen sustento y congruencia con otras fuentes informativas, es decir, la descripción de los acontecimientos es acertada y no desinformada, o sea, no intencionalmente tergiversada como el concepto determina. En la estricta definición académica, los titulares no desinforman, pero unos subinforman más que otros en la versión de los participantes de este acontecimiento político.

Los resultados de la subinformación aquí expuesta coinciden con la opinión pública sobre las manifestaciones desde la perspectiva en China: el $90 \%$ de los chinos continentales rechazan las protestas en Hong Kong (Reikmann, 2019), por la supuesta "única versión" de la historia que se comparte en China. A diferencia de esta explicación, una publicación del SCMP ${ }^{17}$ señala que el rechazo de la población china hacia las manifestaciones no deriva de la versión de los medios de comunicación sino de una conciencia cultural

17. Este medio es identificado por los manifestantes entrevistados como pro-Beijing.

112 México y la Cuenca del Pacífico. Vol. 10, núm. 28 / enero-abril de 2021. 
que procura evitar la violencia (Yi, 2019). Desde esta perspectiva, China no tiene problemas por "falta de información", pues existe la conciencia de que hay algún tipo de censura, ${ }^{18}$ pero también conocen los medios para evadir el muro de fuego digital a través de las VPN ${ }^{19}$ además de que existen múltiples sitios de Internet para informarse, pero el principal problema es la barrera lingüística (Yi, 2019). En este sentido, una de las barreras que promueven la subinformación no provendría de los modos de información de la prensa, sino de una barrera subinformativa individual relacionada con las limitantes lingüísticas para leer en otros idiomas, y ampliar el espectro informativo.

Por otro lado, según el estudio del Centre for Communication and Public Opinion Survey (Lee et al., 2019), el 39\% de los hongkoneses están de acuerdo con los manifestantes radicales, además de que el $70 \%$ está en desacuerdo con el uso de la fuerza policial en contra de estos protestantes. El estudio liderado por Francis Lee de la Chinese Hong Kong Univeristy revela que los propios manifestantes reconocen que las protestas iniciadas en junio tenían la intención de mantenerse pacíficas, pero las manifestaciones radicales son a las que el Gobierno de HK presta atención (Lee et al., 2019). Esto concuerda con el comentario de uno de los manifestantes entrevistados al decir: "Yo no participo en los actos vandálicos, pero... No puedo decir nada, estoy de acuerdo con ellos - protestantes radicales-. De otro modo el Gobierno no quiere escuchar" (Jing, 2019).

Los hallazgos aquí presentados a través del análisis discursivo de los titulares de noticias seleccionados por la importancia de sus fechas en actividades coyunturales de las protestas, coinciden con la opinión pública de ambas regiones; por ende, lo subinformado a través de la prensa refleja y refuerza la opinión pública. Con estos resultados no se pretende caer en un determinismo periodístico, ya que el fenómeno social de informarse es aún más complejo. El "ser informado", según la apreciación de Sartori (2016), requiere un esfuerzo del sujeto individual; sin embargo, los recursos disponibles de información son importantes en la construcción de la opinión pública porque el conocimiento de un acontecer político es el resultado de haber sido informado (Lippman, 2013; Machlup, 1962; Schiller, 1973). La incorporación del concepto subinformación en las teorías de la comunicación evitaría el maniqueísmo información/desinformación, y propone la designación de nuevos

18. La subinformación no es sinónimo de censura, pero puede ser una subcategoría.

19. Virtual Private Network. 
conceptos que describan la complejidad del fenómeno social respecto a los procesos informativos en distintas regiones del mundo, sin dejar de lado las particulares del periodismo de cada zona.

\section{Lecciones para la alfabetización mediática subinformativa}

La subinformación entre la Región Administrativa Especial de Hong Kong y la República Popular China sugiere una pizarra de aprendizaje, pues los resultados de este análisis confirman la estrecha correlación entre la práctica subinformativa de la prensa y parte de la opinión pública. Éstas son algunas lecciones de este primer acercamiento para la alfabetización mediática respecto a la subinformación:

1. La prensa no siempre desinforma, mayormente subinforma. Con el desmoronamiento de la credibilidad de los medios de comunicación masiva tradicionales, existe una tendencia a etiquetarlos como medios de "desinformación" sin atender al marco conceptual de la palabra. Si bien es cierto que hay evidencia de fake news a través de estos medios tradicionales, también la hay en los medios alternativos; por tanto, la confirmación de información a través de la contrastación de fuentes siempre es la mejor recomendación para ambos casos. La alfabetización mediática consiste en comprender que cada medio tendrá una línea editorial que en ocasiones servirá a ciertos intereses políticos como lo estima la teoría instrumentalista del periodismo, pero parte de su contenido sigue los lineamientos éticos de la construcción de la noticia: factualidad e imparcialidad.

2. "Ser informado" conjunta un verbo para convertirse en adjetivo. "Ser informado" requiere un esfuerzo por parte de la audiencia, no es trabajo delegado a una prensa mediática. Independientemente de la cantidad de prensa consultada, es importante reconocer:

a. La naturaleza de la labor periodística subinforma en un primer nivel. La producción y distribución de noticias es compleja y no todo puede ser informado a través de un solo medio; horarios televisivos, espacios en papel, recursos humanos y problemas técnicos en lo digital son algunas de las restricciones que subinforman a priori.

b. Tomar en consideración, al menos, dos líneas editoriales. La lectura de líneas editoriales radicalmente distintas enriquece las perspectivas y marcos referenciales, incluso si la audiencia no simpatiza con ellas. Así también se reconocen las limitantes de contenido de cada prensa. 
c. Definir actores y perspectivas. De este modo será más fácil identificar a quiénes se les da más voz de opinión, y sobre quiénes se subinforma.

d. Leer prensa local e internacional sobre el tema de interés. La prensa internacional tiene puntos de vista en la descripción de un hecho noticioso, que en ocasiones no son percibidos por la prensa local y sus líneas editoriales (véase sugerencia 5).

3. Virar a las plataformas online de prensa libre. Por su naturaleza, la prensa que pertenece a un medio masivo de comunicación está supeditada a las prácticas lucrativas de la "gran agencia", pues no sólo se dedican a producir información sino entretenimiento. Las plataformas de información no lucrativas relativas al periodismo 3.0 comúnmente procuran mantener una línea de periodismo crítico que sirva a la ética primaria del ejercicio periodístico (Pena de Oliveira, 2009). Por tanto, y como este ejercicio comprueba, el periodismo independiente suele mantener un equilibro en la presentación de los acontecimientos, dando voz a la mayoría de los actores involucrados en el acontecer político; aunque generalizar sería erróneo.

4. Participar en los foros de discusión y crear aplicaciones alternativas. Los manifestantes y periodistas de Hong Kong sugieren que uno de los medios más efectivos para adquirir información es a través de foros de discusión online y utilizar menos las redes sociales —en especial evitar Facebook-, pues estos últimos sólo muestran información sugerida para el perfil y no las noticias que les pueden ser útiles. "Estudios muestran que el uso de Facebook para mantenerse informado y comunicado en las manifestaciones es la peor estrategia" (Au, 2019), por ello "es mejor crear las propias fuentes de información, puedes mantenerte informado con las fuentes tradicionales, pero es más importante que todos conozcamos la información que consumimos" (Sheeper, 2019). En consecuencia, la sugerencia es crear y promover canales propios de comunicación a través del espacio digital en el que se discuta la opinión pública después de la lectura de prensa tradicional e independiente.

5. Superar la barrera lingüística. Desde la perspectiva de Ren Yi (2019), pese a la gran muralla de fuego digital en China, existen canales online para obtener información a través de otros medios de lo que de modo superficial se encuentra en la red, de esta manera desde China se tendría la posibilidad de leer medios alternativos en chino o en otros idiomas; sin embargo, esta última variable es de nivel individual. Para los manifestantes no basta con el 
hecho de tener la información escrita en la prensa local de ambas regiones, sino que la prensa china y hongkonesa debería estar escrita en inglés para que "otros países puedan leer lo que sucede" (Sheeper, 2019). Desde esta perspectiva, una situación similar ocurriría en otras regiones del mundo; por ejemplo: de acuerdo con el sitio Internet World Stats (Moreno, 2017), el total de contenido en Internet es en $53.1 \%$ en inglés y sólo un $5 \%$ en español. Esto significa que es posible encontrar contenido de calidad sobre el acontecer local desde la prensa internacional (o, al menos, perspectivas y ejemplos que ayuden a entender realidades locales).

Estas sugerencias reinciden en la importancia de la alfabetización mediática en términos tecnológicos -en la creación de apps y búsqueda de foros de discusión - y en la interpretación de la noticia — análisis de discurso y debate en los foros de discusión-, con el fin de enriquecer el proceso del ser informado en la conciencia de la posición activa de la audiencia. En el proceso de una alfabetización mediática respecto a la desinformación y subinformación, la opinión pública estará constituida por la educación de lectura periodística.

Los hallazgos de este ejercicio y las lecciones sugeridas no pretenden ser declaraciones concluyentes en el estudio de la subinformación, sino proveer el primer acercamiento al campo fértil para la academia en la incorporación de conceptos que diversifiquen la posición maniqueísta información/desinformación sobre el ejercicio periodístico de la prensa tradicional e independiente, adaptada a regiones específicas en relación con la política local y regional.

\section{Referencias}

Aguilar, L. F. V. (2017, Julio - Diciembre). Una reconstucción del concepto de opinión pública. Revista Mexicana de Opinión Pública, 12(23), 125-148. http://www.revistas.unam.mx/index.php/rmop/article/view/60908

$\mathrm{Au}, \mathrm{A}$. (2019, Noviembre 5). S/T. Entrevistadora: Sonia Evangelina Alcántar Jaime.

Blandón, D. R. (2019, Julio 7). Hong Kong: decenas de heridos en nueva jornada violenta de protestas. France 24. https://www.france24.com/es/20190722hong-kong-heridos-protestas-ley-extradicion

Boczkowsky, P. (2006). Digitalizar las noticias: Innovación en los diarios online. Manantial. 
China Central Television (CCTV). (2019, Octubre). 中国中央电视台 [China Central Television]. http://english.cctv.com/

China Daily. (2019, Octubre). China News. http://www.chinadaily.com.cn/ china/

Collins. (2017, noviembre 2). Fake news. Collins 2017 Word of the Year Shortlist. https://www.collinsdictionary.com/word-lovers-blog/new/collins2017-word-of-the-year-shortlist,396,HCB.html

Constitutional and Mainland Affairs Bureau. (1985). Joint Declaration of the Government of the United Kingdom of Great Britain and Northern Ireland and the Government of the People's Republic of China on the Question of Hong Kong. Constitutional and Mainland Affairs Bureau. The Government of the Hong Kong Special Administrative Region of the People's Republic of China. https://www.cmab.gov.hk/en/issues/jd2.htm

Cue, A. M. (2017). China y Hong Kong: a 20 años de su reintegración. El Cotidiano, 32(205), 87-94. https://www.redalyc.org/pdf/325/32553152010.pdf

Díaz, E. R. (1976). Pueblos Subinformados. Monte Ávila Editores.

Fox, C. (1983). Information and Misinformation: An investigation of the notions of information, misinformation, informing and misinforming. Greenwood.

Fraguas de Pablo, M. (1985). Teoría de la Desinformación. Alhambra.

Fraguas de Pablo, M. (2016). La desinformación en la sociedad actual. Cuadernos.Info, (3). https://doi.org/10.7764/cdi.3.887

France 24. (2019, Agosto 12). Las autoridades de Hong Kong cancelan todos los vuelos por protestas dentro del aeropuerto. France 24. https://www.france24. com/es/20190812-manifestantes-bloquo-vuelos-aeropuerto-hong-kong Galdón. G. L. (1994). Desinformación. Método, aspectos y soluciones. EUNSA.

HK Democracy M. (2019, Julio 1). Hong Kong Democracy Movement. HK Democracy M. https://hkrev.info/main_en.php

HKRev - Hong Kong Local News Translation [@HkrevInfo]. (s.f.). Tweets [Twitter profile]. Twitter. https://twitter.com/HkrevInfo

Hong Kong Free Press (HKFP). (2019). Hong Kong Free Press. https://www. hongkongfp.com/

Hong Kong Free Press (HKFP). (2020a). About Hong Kong Free Press. Hong Kong Free Press. https://hongkongfp.com/about/

Hong Kong Free Press (HKFP). (2020b). Support Hong Kong Free Press. Hong Kong Free Press. https://support.hongkongfp.com/

Jacquard, R. (1988). La desinformación: una manipulación del poder. Espasa Calpe. 
Jing. (2019, Octubre 27). S/T. Entrevistadora: Sonia Evangelina Alcántar Jaime.

Lee, F. L. F., Tang, G., Yuen, S., \& Cheng, E. W. (2019, Agosto). Onsite Survey Findings in Hong Kong's Anti-Extradition Bill Protests (Research Report). Centre for Communication and Public Opinion Survey. The Chinese University of Hong Kong. http://www.com.cuhk.edu.hk/ccpos/en/pdf/ ENG_antielab\%20survey\%20public\%20report\%20vf.pdf

Lippmann, W. (2013). La opinión pública (traducción de Blanca Guinea Zubimendi). Cuadernos de Langre. (Libro original publicado en 2003).

Machlup, F. (1962). The Production and Distribution of Knowledge in the United States. Princeton University Press.

Marques de Melo, J. (2009). Pensamiento comunicacional latinoamericano. Entre el saber y el poder. Comunicación Social Ediciones y Publicaciones.

Moreno, G. (2017, Enero 24). La mitad del contenido de Internet está en inglés. Statista. https://es.statista.com/grafico/7736/la-mitad-del-contenidode-internet-esta-en-ingles/

Pena de Oliveira, F. (2009). Teoría del Periodismo. Alfaomega; Comunicación Social.

Ponsonby, A. (1928). Falsehood In Wa-time: Containing an Assortment of Lies Circulated Throughout the Nations During the Great War. Garland Publishing Company.

Radio Television Hong Kong. (2019). 香港電台網站 [Radio Television Hong Kong]. https://www.rthk.hk/

Reikmann, A. (2019, agosto 19). ¿Qué piensan los chinos continentales sobre las protestas en Hong Kong? DW. https://www.dw.com/es/qu\%C3\%A9piensan-los-chinos-continentales-sobre-las-protestas-en-hongkong/a-50083241

Rodríguez, R. (2018). Fundamentos del concepto de desinformación como práctica manipuladora de la comunicación política y las relaciones internacionales. Historia y Comunicación Social 23(1), 231-244. https://doi. org/10.5209/HICS.59843

Sahagún, F. (1998). De Gutenberg a Internet. La Sociedad Internacional de la Información. Diplomacia y Periodismo. Televisión y Guerra. Estudios Internacionales de la Complutense.

Sartori, G. (1993). ¿Qué es la democracia? Editorial Patria.

Sartori, G. (2016). Homo videns. La sociedad teledirigida. Debolsillo.

Schiller, H. (1973). The Mind Managers. Beacon Press. 
Sheeper. (2019, Septiembre-Octubre Varios). S/T. Entrevistadora: Sonia Evangelina Alcántar Jaime. [Correo electrónico y presencial].

South China Morning Post (SCMP). (2019). South China Morning Post. https:// www.scmp.com/

UNESCO. (2018). Journalism, 'Fake News' and Disinformation: A Handbook for Journalism Education and Training. UNESCO. https://unesdoc.unesco. org/ark:/48223/pf0000265552

Vidal, M. L. (2019a, Junio 16). Dos millones de personas inundan Hong Kong para defender las libertades. El País. https://elpais.com/internacional/2019/06/16/actualidad/1560692921_683769.html

Vidal, M. L. (2019b, Octubre 20). Nuevos enfrentamientos entre la policía y manifestantes en Hong Kong. El País. https://elpais.com/internacional/2019/10/20/actualidad/1571582389_900654.html

Westcott, B. (2019, Julio 1). Hong Kong's July 1 protests: What's happened so far. CNN. https://edition.cnn.com/asia/live-news/hong-kong-july-1protests-intl-hnk/h_78fe271ddbfe579d5ad76c0e441d6539

Westerståhl, J. (1983). Objective News Reporting: General Premises. Communication Research, 10(3), 403-424. https://doi. org $/ 10.1177 / 009365083010003007$

Xinhua News Agency. (2019). 新华通讯 [Xinhua News Agency]. http://www. xinhuanet.com/

Yi, R. (2019, Octubre 25). Mainland Chinese who oppose Hong Kong's protests aren't brainwashed by censorship despite what the West might think. South China Morning Post. https://www.scmp.com/comment/opinion/ article/3034211/mainland-chinese-who-oppose-hong-kongs-protestsarent-brainwashed 